\title{
Assessment of Anticancer Drug Effects on Pancreatic Cancer Cells under Glucose-Depleted Conditions Using Intracellular and Extracellular Amino Acid Metabolomics
}

\author{
Ryoko Tomita, ${ }^{a}$ Kenichiro Todoroki, ${ }^{b}$ Tadashi Hayama, ${ }^{a}$ Hideyuki Yoshida, ${ }^{a}$ Toshihiro Fujioka, ${ }^{a}$ \\ Manabu Nakashima, ${ }^{a}$ Masatoshi Yamaguchi, ${ }^{a}$ and Hitoshi Nohta*,a \\ ${ }^{a}$ Faculty of Pharmaceutical Sciences, Fukuoka University; 8-19-1 Nanakuma, Johnan-ku, Fukuoka 814-0180, \\ Japan: and ${ }^{b}$ Laboratory of Analytical and Bio-Analytical Chemistry, School of Pharmaceutical Sciences, University \\ of Shizuoka; 52-1 Yada, Suruga-ku, Shizuoka 422-8526, Japan. \\ Received September 15, 2017; accepted November 5, 2017
}

\begin{abstract}
Previously, we developed a method to evaluate states of cells treated with anticancer drugs via the comprehensive analysis of amino acids, termed amino acid metabolomics. In the present study, we evaluated the effects of the anticancer drugs, gemcitabine hydrochloride and pyrvinium pamoate, on the proliferation of a pancreatic cancer cell line (PANC-1) under hypoglycemic conditions using amino acid metabolomics. Intracellular and extracellular amino acid profiles of PANC-1 were determined by hydrophilic interaction chromatography-tandem mass spectrometry with simple pretreatment. Changes to the drugs' anticancer effects resulting from glucose starvation conditions were presented in score plots obtained from principal component analyses. In particular, the analysis of intracellular amino acids was found to be the superior approach because the results allowed a clearer assessment of the cell state. Further, orthogonal partial least squares discriminant analysis was performed to search for amino acid candidates that discriminate with anticancer drug-treated PANC-1 cells. We identified several amino acids that might be able to distinguish the drugtreated group from the control group. These results might provide a better understanding of the mechanisms underlying cell responses such as drug resistance or austerity. The present study is the first to evaluate the efficacy of anticancer drugs under glucose starvation based on the analysis of the variation of extracellular and intracellular amino acid profiles in vitro.
\end{abstract}

Key words amino acid metabolomics; pancreatic cancer cell; glucose depletion; biomarker candidate

Metabolomics is a comprehensive analysis of numerous small endogenous molecules contained in cells, biological fluids, and tissues. In addition to a complementary understanding of physiological functions, metabolomics is also a promising tool to explore potential biomarkers for diagnosis, prognosis, and therapeutic response. ${ }^{1)}$ It has proven to be very useful not only in medical and life sciences, but also in clinical research, ${ }^{2,3)}$ drug discovery, ${ }^{4)}$ environmental toxicology, ${ }^{5}$ and food science. ${ }^{6,7)}$ Recently, among the available focused metabolomics approaches, amino acid metabolomics analysis, which focuses on amino acids as key metabolites, such as aminoindex $^{8,9)}$ or chiral amino acid metabolomics, ${ }^{10-12)}$ has received considerable attention because of its utility in the diagnosis and monitoring of pathological conditions. Exogenous and endogenous amino acids are hub substances in regulated metabolic networks, and are also relatively abundant in many biological samples. Therefore, they are thought to have superior properties as targets of focused metabolomics. In several studies, alterations in the free amino acid profile were found to be related to various disease states, such as cancer. ${ }^{13,14)}$ Previously, we evaluated the effect of several anticancer agents on cancer cell proliferation using amino acid metabolomics analysis of the culture medium. Amino acid measurement was performed using fluorescence derivatization with 6-aminoquinolyl- $N$-hydroxysuccinimidyl carbamate, followed by HPLC analysis. ${ }^{15)}$ The method is adequate to determine amino acid levels with high sensitivity and reproducibility. We could evaluate the efficacy of several anticancer drugs to colorectal adenocarcinoma cells (colo201) at the cultured cell level. At the same time, we identified amino acid candidates for therapeutic biomarkers of anticancer drugs. Furthermore, hydrophilic interaction chromatography-tandem mass spectrometry (LC-MS/MS) analysis allows simple analysis without tedious and time-consuming derivatization procedures. ${ }^{16)}$ The alteration of cellular metabolism under a simulated tumor microenvironment was demonstrated, and characteristic marker candidates for tumor microenvironment conditions of colorectal adenocarcinoma cell line (DLD-1) were identified. Both methods enable reliable metabolomics analysis. Therefore, in this study, PANC-1, derived from human pancreatic cancer, was used as a model cell line under glucose starvation conditions to evaluate the effects of gemcitabine hydrochloride (GEM) and pyrvinium pamoate (PP). Cancer cells exposed to a tumor microenvironment, such as hypoxia or nutrient starvation, promote energy production by anaerobic glycolysis to survive and proliferate. ${ }^{17,18)}$ Changes to the efficacy of the anticancer drugs dependent on the glucose concentration were determined by analyzing extracellular amino acid profiles. However, it is sometimes difficult to determine slight alterations in amino acid levels caused by different cancer cell states because many measured amino acids are abundant in the culture medium and in biological fluids. Therefore, amino acid metabolomics analysis was performed not only on culture medium, but also on cell extracts to evaluate their differences (Fig. 1). PANC-1 cells were cultured with either GEM or PP under normoglycemic and hypoglycemic conditions, and the culture media were collected and analyzed by LC-MS/MS after a deproteinization. In contrast, intracellular metabolites were analyzed after 


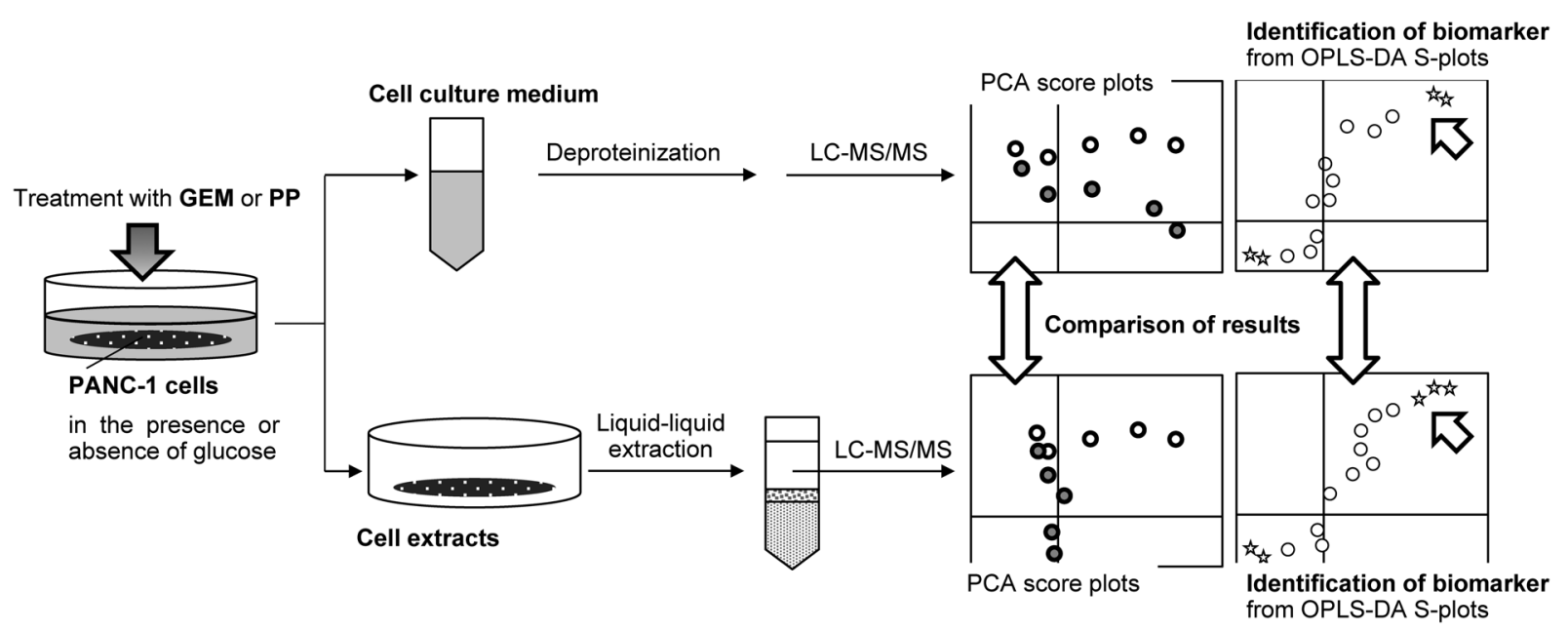

Fig. 1. Schematic Flow of Amino Acid Metabolomics Study of PANC-1 Culture Medium and Cell Extracts

GEM, gemcitabine hydrochloride; PP, pyrvinium pamoate; PCA, principal component analysis; OPLS-DA, orthogonal partial least squares discriminant analysis.

simple extraction procedure. Intracellular and extracellular amino acid profiles derived from the same cancer cells were compared, and candidate biomarkers of anticancer drugs' effects were identified. GEM is classified as an antimetabolite and is commonly used for systemic chemotherapy of pancreatic cancer. After entry into cells via a nucleotide active transporter and phosphorylation by a kinase, the diphosphate form of the drug potently inhibits ribonucleotide reductase, and the triphosphate form inhibits DNA synthesis by competitive incorporation into DNA. ${ }^{19,20)}$ Although GEM has been used as standard therapeutic agent against solid cancers, it was recently reported that cancer cells become resistant to conventional chemotherapy under tumor microenvironment conditions, which is characterized by nutrient and oxygen deficiencies. ${ }^{17)}$ Therefore, anticancer therapies targeting cancer cells in tumor microenvironment conditions are the focus of new therapeutic strategies. For example, under normal culture conditions, electrons generated in the tricarboxylic acid (TCA) cycle participate in the production of ATP, mainly via the aerobic respiratory chain. PP, used clinically as an anthelminthic, is not cytotoxic because of the enhancement of succinatequinone reductase activity, regardless of the inhibition of complex-I activity by $\mathrm{PP}^{21)}$ On the contrary, in mitochondria of cancer cells under hypoxic and glucose-deficient conditions that mimic the tumor microenvironment, the respiratory chain plays a role in energy production by shifting from aerobic to anaerobic activity. PP selectively inhibits the reduced nicotinamide adenine dinucleotide (NADH)-fumarate reductase in the respiratory chain. ${ }^{22-24)}$ Amino acids are thought to be used instead of glucose in the NADH-fumarate reductase system because aspartic acid is converted into fumarate. The results obtained provide useful information that will improve our understanding of the metabolic characteristics of cancer cells.

\section{MATERIALS AND METHODS}

Materials and Chemicals The standard amino acid mixture solution type $\mathrm{H}$ [2.5 mM each of L-aspartic acid (Asp), L-serine (Ser), L-glutamic acid (Glu), glycine (Gly), L-histidine (His), L-arginine (Arg), L-threonine (Thr), L-alanine (Ala), L-proline (Pro), L-tyrosine (Tyr), L-valine (Val), L-methionine (Met), L-lysine (Lys), L-isoleucine (Ile), L-leucine (Leu), L- phenylalanine (Phe), and L-cystine ((Cys) $\left.\left.)_{2}\right)\right]$, LC-grade acetonitrile, LC-grade methanol, and dimethyl sulfoxide (DMSO) were purchased from Wako Pure Chemical Industries, Ltd. (Osaka, Japan). Distilled water, LC-grade tetrahydrofuran (THF), formic acid, chloroform, phosphate-buffered saline (PBS), glutamine (Gln) solution, sodium pyruvate solution, and non-essential amino acids solution were obtained from Nacalai Tesque (Kyoto, Japan). Asparagine (Asn) monohydrate, Gln, tryptophan (Trp), $\alpha$-aminobutyric acid, and GEM were purchased from Tokyo Chemical Industry (Tokyo, Japan). Dulbecco's modified Eagle's medium (DMEM) containing $25 \mathrm{~mm}$ glucose, glucose-free DMEM, dialyzed fetal bovine serum (FBS), penicillin streptomycin, and a $0.4 \%$ trypan blue solution were purchased from Gibco (Auckland, New Zealand). Ammonium formate was purchased from Kanto Chemical (Tokyo, Japan). Cell counting kit-8 containing the tetrazolium salt (WST-8) reagent was obtained from Dojindo Laboratories (Kumamoto, Japan). PP was purchased from Sigma-Aldrich (St. Louis, MO, U.S.A.). Stable isotope-labeled amino acids, including L-Glu- $d_{5}$, L-His- $d_{3}$, L-Lys- $d_{8}$, L-Phe- $d_{5}$, and $\mathrm{L}-\mathrm{Val}-d_{8}$, were purchased from CDN Isotopes (PointeClaire, Quebec, Canada).

Evaluation of PANC-1 Cell Viability Affected by GEM and PP The human pancreatic adenocarcinoma cell line PANC-1 was obtained from the American Type Culture Collection (Manassas, VA, U.S.A.). PANC-1 cells were kept in high glucose DMEM with $10 \%$ dialyzed FBS, $1 \%$ penicillin streptomycin, $0.1 \mathrm{~mm}$ non-essential amino acids solution, $2 \mathrm{~mm}$ Gln solution, and $1 \mathrm{~mm}$ sodium pyruvate solution at $37^{\circ} \mathrm{C}$ under $5 \% \mathrm{CO}_{2}$. Cells in their exponential growth phase $(80 \%$ confluency in the flask) were used in the experiments. Under hypoglycemic conditions, glucose-free DMEM was used instead of high glucose DMEM.

To determine the $\mathrm{IC}_{50}$ values of GEM and PP, the cell viability was calculated using the cell counting kit- 8 or the trypan blue exclusion methods. First, cells were seeded in $100 \mu \mathrm{L}$ of culture medium $\left(2.5 \times 10^{4}\right.$ cells $\left./ \mathrm{mL}\right)$. After $24 \mathrm{~h}$, cells were washed twice with PBS and exposed for 96h to GEM (from 1.25 to $10 \mu \mathrm{M}$ ) under normoglycemic conditions and to PP (from 12.5 to $100 \mathrm{~nm}$ ) under hypoglycemic conditions. For the cell counting kit- 8 assay, cells were treated with $10 \mu \mathrm{L}$ of WST- 8 reagent solution for the last $4 \mathrm{~h}$ of incubation. Absor- 
bance was measured at $450 \mathrm{~nm}$ using a Sunrise rainbow RC-R microplate reader (TECAN, Männedorf, Switzerland). For trypan blue staining, cells were washed twice with PBS after removal of the medium and collected. An aliquot of the cell suspension was added to an equal volume of a $0.4 \%$ trypan blue solution and viable cells were counted with a hemocytometer. The $\mathrm{IC}_{50}$ values were calculated from each doseresponse curve. Moreover, cells were also incubated from 0 to $96 \mathrm{~h}$ with GEM and PP at adequate concentrations under both normoglycemic and hypoglycemic conditions to confirm the efficacy of the anticancer drugs. Cells cultured under each condition without drug-treatment were used as controls.

Measurement of Extracellular and Intracellular Amino Acid Concentrations For extracellular amino acid metabolomics, cells $\left(2.5 \times 10^{4}\right.$ cells $\left./ \mathrm{mL}\right)$ were seeded in $100 \mu \mathrm{L}$ of culture medium in 96-well plates. The medium was then changed to high glucose or glucose-free medium containing each drug at the arbitrary concentration, and the cells were incubated for up to $96 \mathrm{~h}$. After $0,24,48,72,84$, and $96 \mathrm{~h}$, an aliquot of each culture medium of the drug-treated and control samples was deproteinized with ice-cold methanol and injected into the LC apparatus, as previously described. ${ }^{16)}$

Alternatively, cells were seeded in $1 \mathrm{~mL}$ of culture medium in 24-well plates and pre-incubated for intracellular amino acid metabolomics. The medium was changed and the cells were exposed to the anticancer drugs at their $\mathrm{IC}_{50}$ values for $96 \mathrm{~h}$, as described above. After the indicated incubation period, the culture medium was removed from each well, and the cells were washed twice with PBS. Cells were then treated with $500 \mu \mathrm{L}$ of methanol for $5 \mathrm{~min}$ and $250 \mu \mathrm{L}$ of distilled water containing an internal standard solution $(0.4 \mathrm{~mm}$ each of Glu- $d_{5}$, His- $d_{3}$, Lys- $d_{8}$, Phe- $d_{5}$, and Val- $d_{8}$ ) in each well. The solution was mixed and transferred into $2.0-\mathrm{mL}$ tubes, and then distilled water $(250 \mu \mathrm{L})$ and chloroform $(500 \mu \mathrm{L})$ were added. After vigorous mixing, the upper aqueous layer containing hydrophilic metabolites was obtained by centrifugation (at $20000 \times \boldsymbol{g}$ for $10 \mathrm{~min}$ at $4^{\circ} \mathrm{C}$ ) and transferred into a $1.5-\mathrm{mL}$ tube. The supernatant was concentrated in vacuo, redissolved in $2 \%$ formic acid, and injected into the LC apparatus.

Extracellular and intracellular free amino acids were analyzed by LC-MS/MS $(n=3)$. The LC-MS/MS conditions were as previously described. ${ }^{16)}$

Statistical Analysis The sequential amino acid concentra-
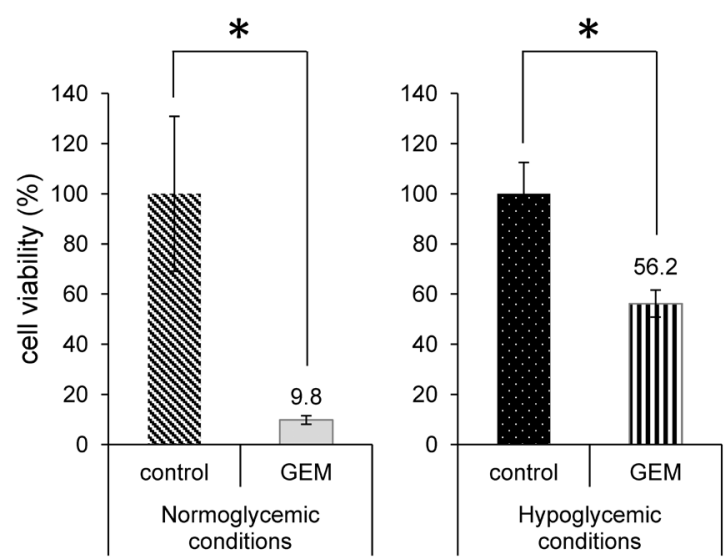

tions obtained from each sample using LC-MS/MS analysis were imported as variables into the SIMCA-P+ software version 12.0 (Umetrics, Umeå, Sweden). For statistical multivariate analysis, principal component analysis (PCA) and cluster analysis were performed as previously described. ${ }^{15,16)}$ Orthogonal partial least squares discriminant analysis (OPLS-DA) was used to explore potential class-discrimination markers, and amino acids that mainly contributed to the classification were identified in S-plots generated using OPLS-DA.

\section{RESULTS}

Viability of PANC-1 Cells Treated with GEM and PP We determined the viability of PANC-1 cells treated with GEM under normoglycemic conditions or PP under hypoglycemic conditions using the cell counting kit- 8 assay. The $\mathrm{IC}_{50}$ values of GEM and PP were approximately 1 and $0.05 \mu \mathrm{M}$, respectively. Because the cell counting kit- 8 assay detects variations of the NADH-reductase activity ${ }^{25)}$ and PP also inhibits the NADH-reductase, the $\mathrm{IC}_{50}$ value of PP measured by the cell counting kit- 8 assay might be affected by the metabolic alterations. Therefore, we decided to determine the $\mathrm{IC}_{50}$ value of PP under hypoglycemic conditions also by trypan blue staining, through which obtained the value of approximately $0.02 \mu \mathrm{M}$. Although, we obtained two different $\mathrm{IC}_{50}$ values of PP with two methods, we could confirm that PANC-1 cell growth is strongly inhibited upon treatment with $0.05 \mu \mathrm{M}$ PP. Next, we determined the viability of PANC-1 cells cultured for $96 \mathrm{~h}$ under both normoglycemic and hypoglycemic conditions with either GEM $(1 \mu \mathrm{M})$ or PP $(0.05 \mu \mathrm{M})$ by trypan blue staining (Fig. 2). Under normoglycemic conditions, the cell viability of PANC-1 cells treated with GEM markedly decreased to approximately $10 \%$ of the control. On the other hand, approximately $55 \%$ of the cells cultured under hypoglycemic conditions and treated with GEM were alive after $96 \mathrm{~h}$ of treatment, showing a reduction of GEM anticancer activity in hypoglycemic conditions. Nutrient deprivation causes a delay in cell cycle progression and often results in G1 arrest. $^{26)}$ It has also been reported that heat shock proteins (HSPs) are induced and the anti-apoptotic pathway is activated by nutrient starvation in cancer cells. ${ }^{27,28)}$ Thus, it is not unexpected that these anticancer drugs show reduced anticancer activity under hypoglycemic conditions, although
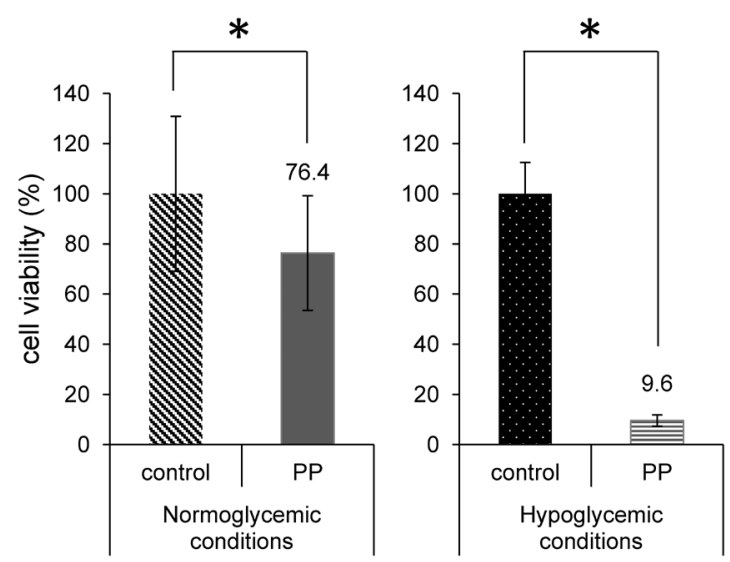

Fig. 2. Relative Cell Viability of Drug-Treated PANC-1 Cells under Normoglycemic and Hypoglycemic Conditions

PANC-1 cells were treated with $1 \mu \mathrm{M}$ GEM or $0.05 \mu \mathrm{M}$ PP under normoglycemic or hypoglycemic conditions for $96 \mathrm{~h}$. Cell viability is shown as a percentage of the control under each condition. Data represent the mean \pm S.D. $(n=5)$. The $p$ values were evaluated by student $t$-test $(*: p<0.05)$. 
different mechanisms might be at play among different cell lines and different drugs. The cell viability of PANC-1 cells treated with PP under normoglycemic conditions decreased to approximately $75 \%$ of the control, whereas the cell viability of PP-treated PANC-1 cells under hypoglycemic conditions was markedly reduced to approximately $10 \%$ compared with the control. These results showed that PP is more effective under hypoglycemic conditions, which is consistent with the results of previous studies. ${ }^{21-24)}$ To evaluate the amino acid metabolic alterations upon these treatments, PANC-1 cells, cultured as described above, were analyzed by amino acid metabolomics.

PCA of PANC-1 Cells Treated with GEM under Normoglycemic and Hypoglycemic Conditions The intracellular and extracellular amino acid contents of PANC-1 cells cultured for $96 \mathrm{~h}$ with GEM under normoglycemic and hypoglycemic conditions were determined using LC-MS/MS analysis. Figure 3A shows the PCA score plot obtained from extracellular amino acid analysis of PANC-1 treated with or without GEM under normoglycemic conditions. The sum of the contributions of principal component 1 (PC1) and 2 (PC2) was $68.7 \%$, which retained sufficient information from the original data. In the score plot, samples incubated briefly were located close to each other, regardless of GEM treatment. As the incubation time increased, especially in samples incubated for 84 and $96 \mathrm{~h}$, the coordinate positions were separated and clustered as GEM-treated and untreated groups by cluster analysis (Fig. 3A). These results indicated that the effects of GEM on PANC-1 cells are reflected in the differences in coordinate positions. The score plot formed by $\mathrm{PC} 1$ and $\mathrm{PC} 2$ obtained from PCA of extracellular amino acids, when treated with or without GEM under hypoglycemic conditions, also accounted for $71.3 \%$ of the variance in the data (Fig. 3B). Although the coordinate positions reflected the trend of the data with sufficient contribution ratios, samples incubated for the same period of time were close to each other on the plot. The grouping of GEM-treated and control cells reflected the suppression of GEM anticancer effects on PANC-1 cells under hypoglycemic conditions, as shown in the cell viability experiments (Fig. 2). PCA score plots obtained from analyses of intracellular amino acids of PANC-1, when treated with or without GEM under either normoglycemic or hypoglycemic conditions are shown in Figs. 3C and D, respectively. Under normoglycemic conditions, two coordinates (PC1 and $\mathrm{PC} 2$ ) accounted for $95.3 \%$ of the variance and could discriminate between GEM-treated and control groups (Fig. 3C). Samples incubated for over $72 \mathrm{~h}$ with or without GEM were classified as separate clusters (GEM-treated and control groups) by cluster analysis. The coordinate positions were very far apart; therefore, the two groups were more clearly discriminated by comparing the plot obtained from the analysis of extracellular amino acids shown above (Fig. 3A). In contrast, the score plot obtained from PCA of the intracellular amino acid content, for cells treated with or without GEM under hypoglycemic conditions, could not discriminate the GEM treatments (Fig. 3D).

The results showed that our proposed amino acid metabolomics method is useful to discriminate the efficacy of GEM using either the intracellular or extracellular amino acid content (Figs. 3A, C). Next, the amino acids that contributed to the classification of the groups were identified from the loading plots in parallel to each score plot under normoglycemic conditions. In the loading plot obtained from the extracellular amino acid analysis, most amino acids (variables) were spread
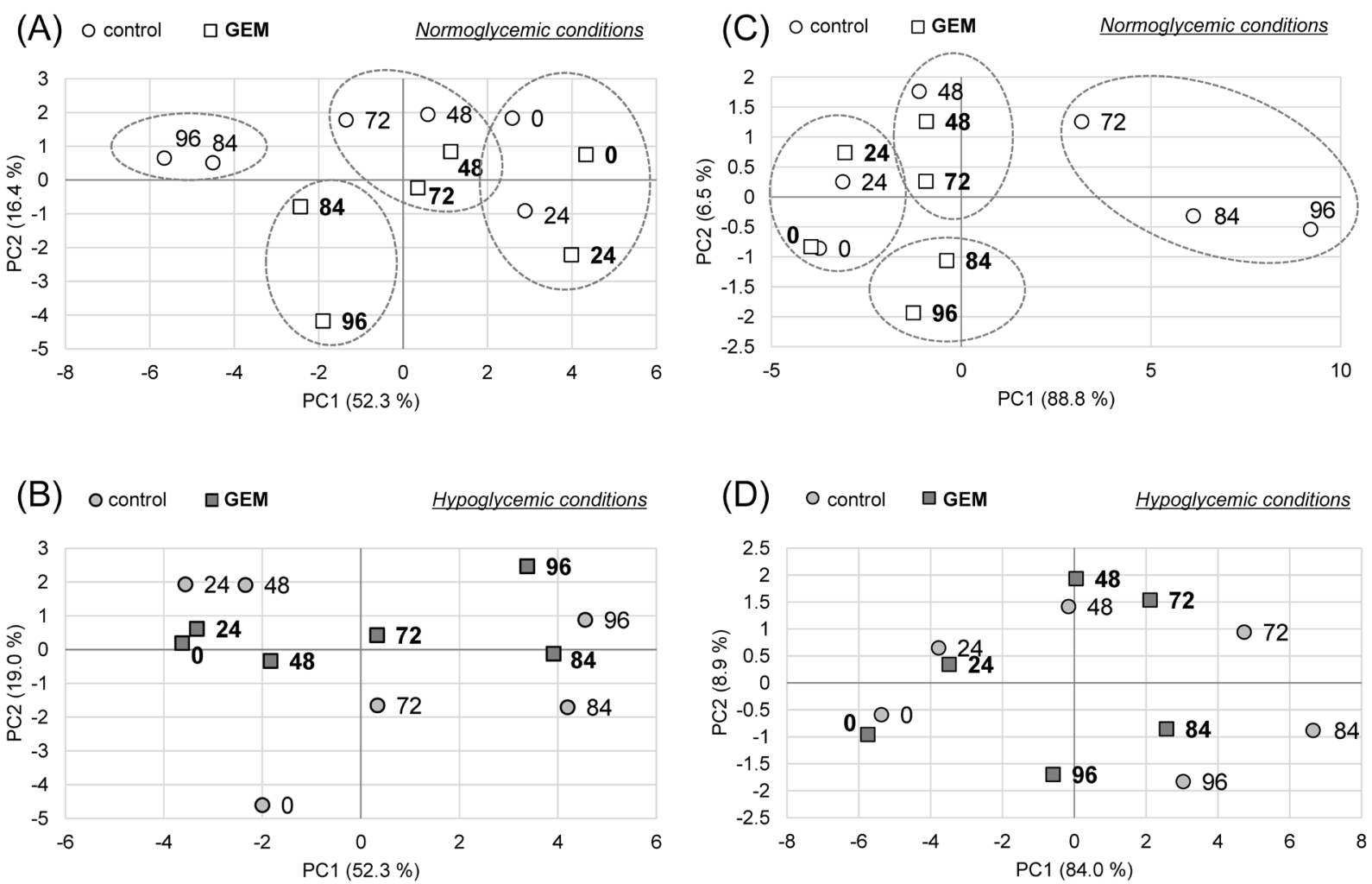

Fig. 3. Score Plots Obtained from Analyses of Extracellular (A and B) and Intracellular (C and D) Amino Acids of PANC-1 Cells Treated with GEM under Either Normoglycemic (Upper Row) or Hypoglycemic (Bottom Row) Conditions

PC1 and PC2 represent principal components 1 and 2, respectively. 
(A)

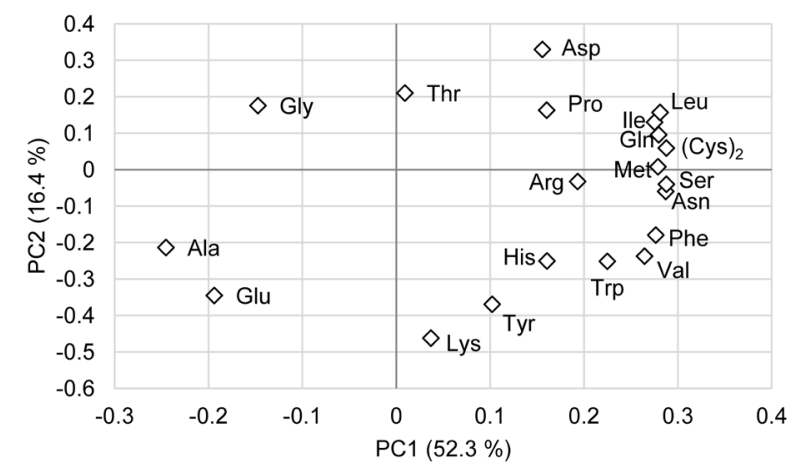

(B)

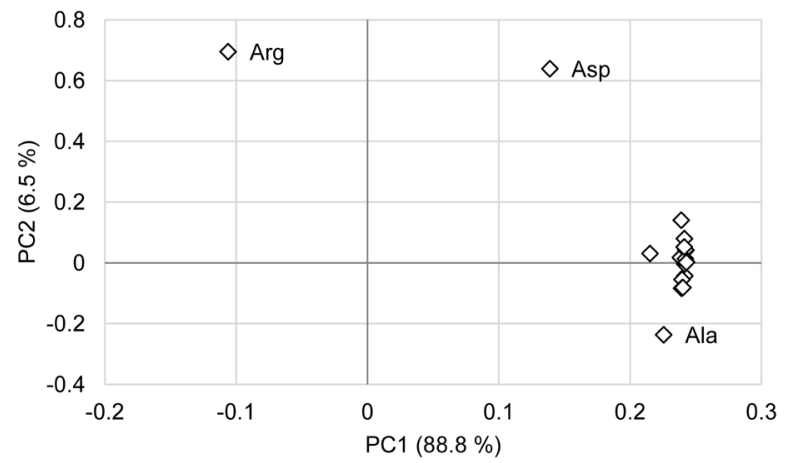

Fig. 4. Loading Plots Obtained from Analyses of Extracellular (A) and Intracellular (B) Amino Acids of PANC-1 Cells Treated with GEM under Normoglycemic Conditions

PC1 and PC2 represent principal components 1 and 2, respectively.

widely (Fig. 4A). The results suggested that the amino acids contributed almost equally to determine the coordinate position in the score plots; no amino acids that might distinguish anticancer effects were observed. In contrast, the PC1 coordinate in the score plot obtained from intracellular amino acid analysis reflected the proliferation process of PANC-1 cells under normoglycemic conditions. The PC1 scores (coordinate position on the horizontal axis) of the score plot differed greatly between both groups because PANC-1 cell growth was suppressed upon GEM treatment. In brief, there is significant information on the $\mathrm{PC} 1$, but not the $\mathrm{PC} 2$, axis because the contribution of $\mathrm{PC} 1$ to discriminate between GEM and control groups was greater. The loading plot obtained from analysis of the intracellular amino acids showed that most amino acids were on the right side of the horizontal axis (PC1), and Arg, located diagonally, contributed to the group classification (Fig. $4 \mathrm{~B})$.

PCA of PANC-1 Cells Treated with PP under Normoglycemic and Hypoglycemic Conditions The intracellular and extracellular amino acid contents of PANC-1 cells cultured for $96 \mathrm{~h}$ with PP under normoglycemic and hypoglycemic conditions were determined using LC-MS/MS analysis. Score plots obtained from the extracellular amino acid analysis were compared under normoglycemic and hypoglycemic conditions (Figs. 5A, B). In the score plots, the sum of the contributions of the two principal components under normoglycemic and
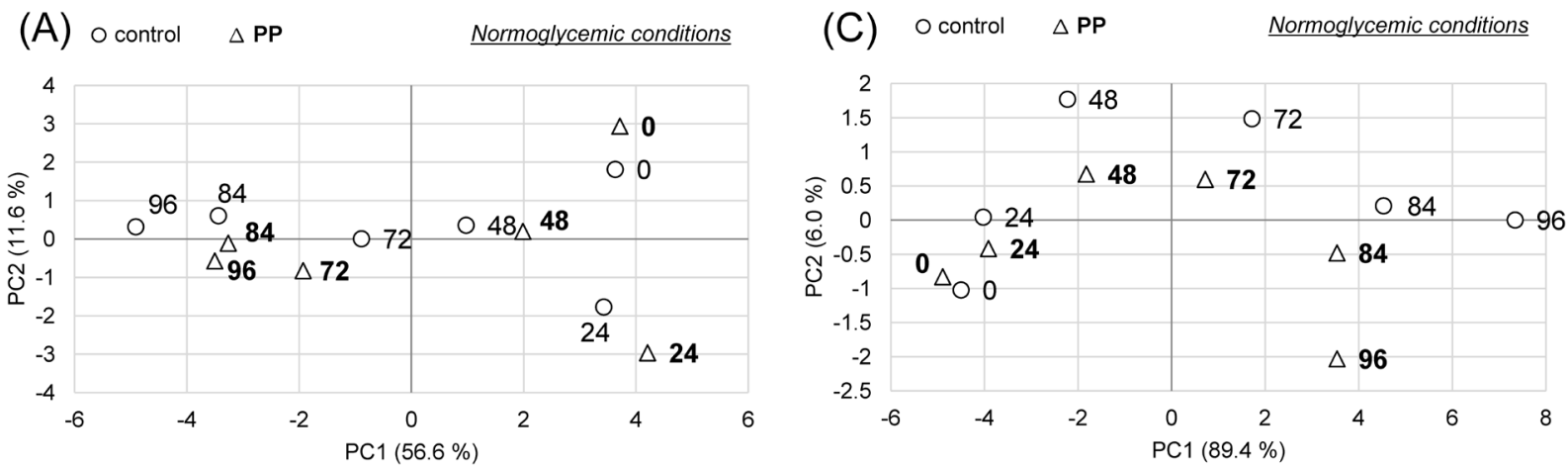

(B) 0 control $\triangle \mathrm{PP}$ Hypoglycemic conditions

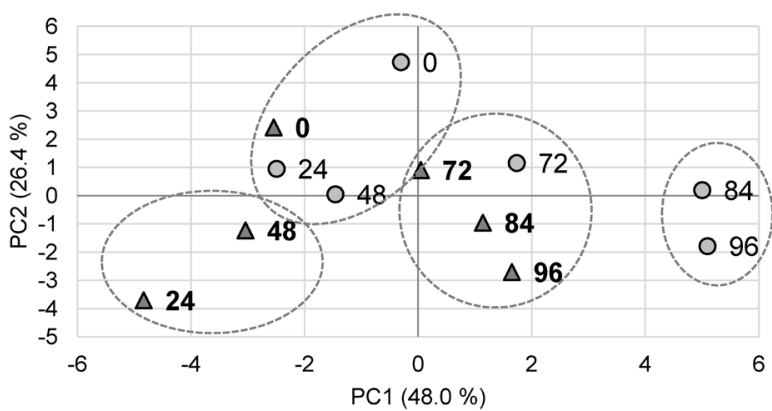

(D) O control $\triangle \mathrm{PP}$ Hypoglycemic conditions

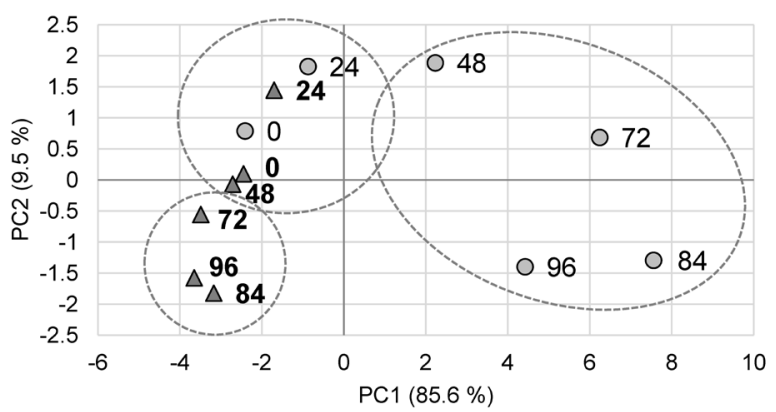

Fig. 5. Score Plots Obtained from Analyses of Extracellular (A and B) and Intracellular (C and D) Amino Acids of PANC-1 Cells Treated with PP under Either Normoglycemic (Upper Row) or Hypoglycemic (Bottom Row) Conditions

$\mathrm{PC} 1$ and PC2 represent principal components 1 and 2, respectively. 
(A)

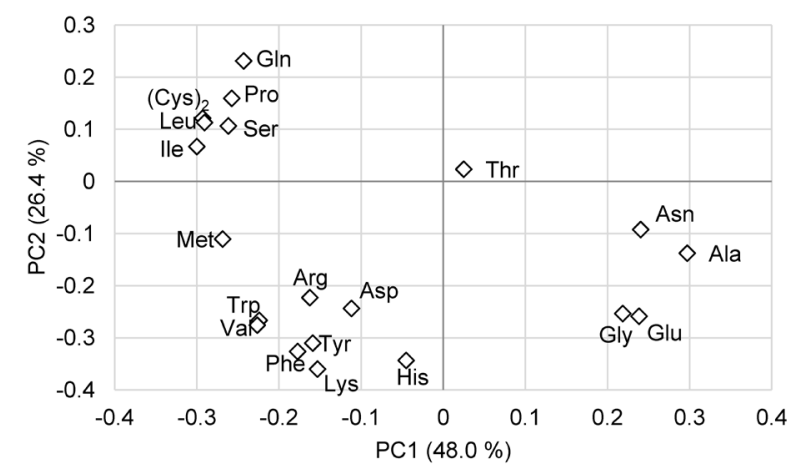

(B)

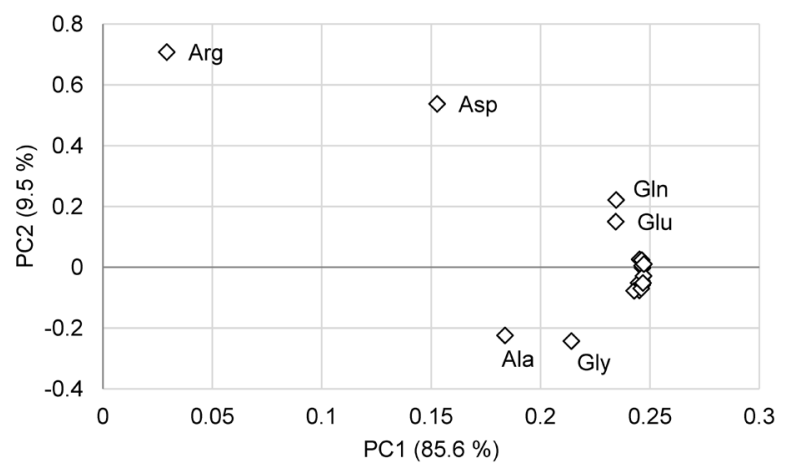

Fig. 6. Loading Plots Obtained from Analyses of Extracellular (A) and Intracellular (B) Amino Acids of PANC-1 Cells Treated with PP under Hypoglycemic Conditions

PC1 and PC2 represent principal components 1 and 2, respectively.

hypoglycemic conditions were 68.2 and $74.4 \%$, respectively, and both contained sufficient information from the original data. Comparison of the score plots showed that samples subjected to the same cultivation period under normoglycemic conditions were close to each other on the plots, regardless of the PP treatment (Fig. 5A). However, samples incubated for 84 and $96 \mathrm{~h}$ with PP under hypoglycemic conditions could be discriminated from the control samples (Fig. 5B). Thus, the PPtreated group was discriminated from the control group by the PC1 scores only under hypoglycemic conditions. However, the separation was not complete, because the coordinate positions on the plot were not very distant from each other. Similarly, the PCA score plots obtained from the intracellular amino acid analysis, when cells were treated with PP under either normoglycemic or hypoglycemic conditions, were compared. As shown in Fig. 5C, samples treated with PP under normoglycemic conditions, with the exception of samples incubated for $96 \mathrm{~h}$, were not discriminated from the controls in the score plot. With decreasing glucose levels owing to the long cultivation time, PP might have a weak effect against PANC-1 cells after $96 \mathrm{~h}$, even in normoglycemic conditions, as shown in the cell viability experiments (Fig. 2). In the PCA score plot (Fig. 5D), samples treated with PP under hypoglycemic conditions were distinguished from the controls by differences in the $\mathrm{PC} 1$ scores. Comparing these results to the score plot obtained from analysis of the extracellular amino acids (Fig. 5B), a clear discrimination was achieved by PC1 only for samples incubated for over $48 \mathrm{~h}$ under hypoglycemic conditions. These results showed that our proposed method could also confirm the selectivity of PP to glucose starvation, as seen in Fig. 2.

The candidate amino acids contributing to group classification were investigated using loading plots corresponding to each score plot (Fig. 6). The results from the extracellular amino acid analysis of PANC-1 under hypoglycemic conditions showed that almost amino acids, except Thr, were scattered throughout the plot (Fig. 6A) because the PP-treated samples were not fully distinguished from the controls in the score plot. Therefore, we inferred that there is no interesting variable that correlates with PP treatment. In contrast, $\mathrm{PCl}$ allowed the discrimination of samples belonging to the PP-treated group in the score plot obtained from the analysis of the intracellular amino acids. Thus, most amino acids with a high coefficient on the PCl axis (far from the plot center) were considered to contribute equally to the discrimination of PP treatment. Therefore, most amino acids, including Arg, contributed to the discrimination of PP treatment (Fig. 6B), which prevented the identification of interesting variables that correlated with this treatment.

Searching for Amino Acid Candidates to Evaluate the Efficacy of Anticancer Drugs The effects of anticancer drugs could be estimated because different amino acid profiles were observed between anticancer drug-treated and control groups in either analysis of the extracellular and intracellular amino acids content. However, there were no variables contributing to the discrimination of groups in any score plots. Therefore, we decided to analyze our data using a different statistical method. We used OPLS-DA using class information of data as variables, in addition to amino acid content data, instead of PCA. The analysis was performed using only samples considered to be different from the control in each score plot. From the S-plots obtained, we selected amino acid candidates that might allow the evaluation of the efficacy of anticancer drugs. As shown in Fig. 7A, many variables (for example Glu, Ile, Leu, Met, Asn, and Pro) were considered as candidate markers to discriminate GEM efficacy under normoglycemic conditions in the S-plot obtained from the analysis of extracellular amino acids when cultured for 84 and $96 \mathrm{~h}$. The concentrations of these variables in samples treated with GEM for $96 \mathrm{~h}$ and compared to controls were as follows: Glu, $\times 2.5$; Ile, $\times 0.6$; Leu, $\times 0.5$; Met, $\times 0.6$; Asn, $\times 0.7$; and Pro, $\times 1.2$. In addition, four variables (Glu, $\times 0.2$; Trp, $\times 0.3$; Tyr, $\times 0.2$; and Gln, $\times 0.4$ compared to controls, after treatment for $96 \mathrm{~h}$ ) were identified in the S-plot obtained from the analysis of intracellular amino acids when cells were cultured with GEM for more than $72 \mathrm{~h}$ (Fig. 7C). By contrast, many variables (Ser, $\times 1.8$; Val, $\times 0.1 ;(\mathrm{Cys})_{2}, \times 1.6$; His, $\times 1.1$; Glu, $\times 0.8$; Asn, $\times 0.8$; and Ala, $\times 0.8$ compared to controls, after treatment for $96 \mathrm{~h})$ were identified as contributing variables to discriminate PP treatment under hypoglycemic conditions in the S-plot obtained from the analysis of extracellular amino acids at 84 and $96 \mathrm{~h}$ (Fig. 7B). Similarly, three variables (Gln, $\times 0.2$; Met, $\times 0.1$; and Ser, $\times 0.1$ compared to controls, after treatment for 96h) were identified as indices of discrimination for PP treatment in the S-plot obtained from the analysis of intracellular amino acids when the cells were cultivated for more than $48 \mathrm{~h}$ (Fig. 7D). Previous studies reported that the concentration of several amino acids in the plasma is significantly altered in 
(A) PANC-1 cultured for 84 and $96 \mathrm{~h}$

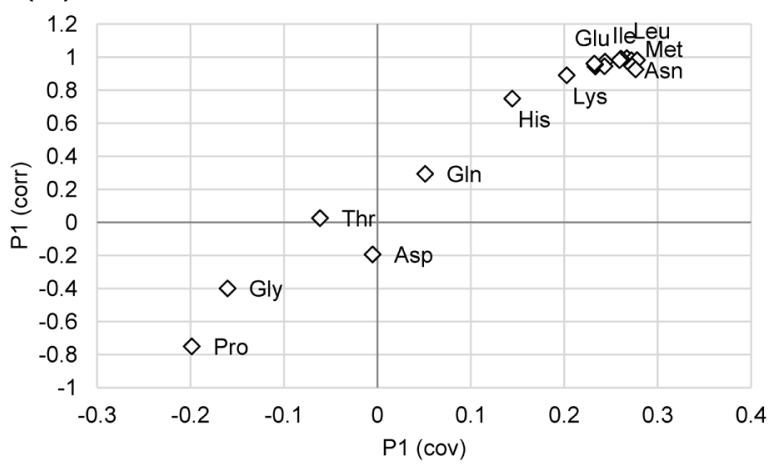

\section{(B) PANC-1 cultured for 84 and $96 \mathrm{~h}$}

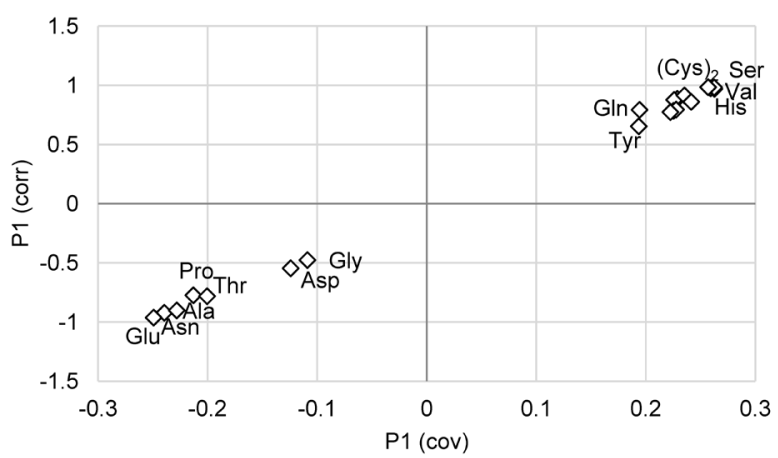

(C) PANC-1 cultured for 72,84 , and $96 \mathrm{~h}$

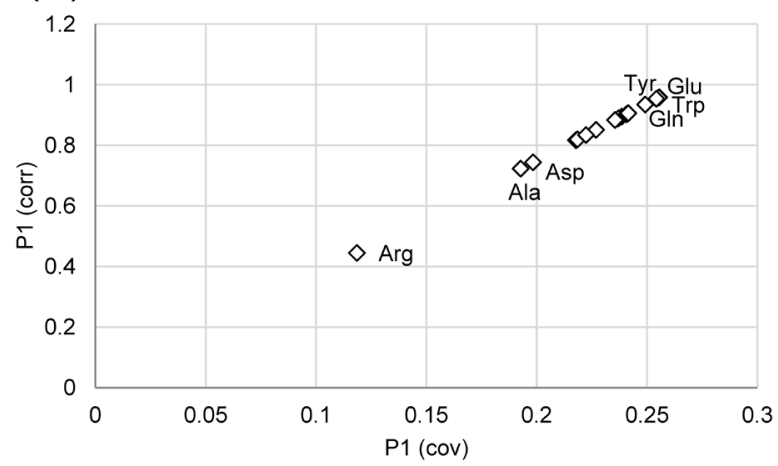

(D) PANC-1 cultured for $48,72,84$, and $96 \mathrm{~h}$

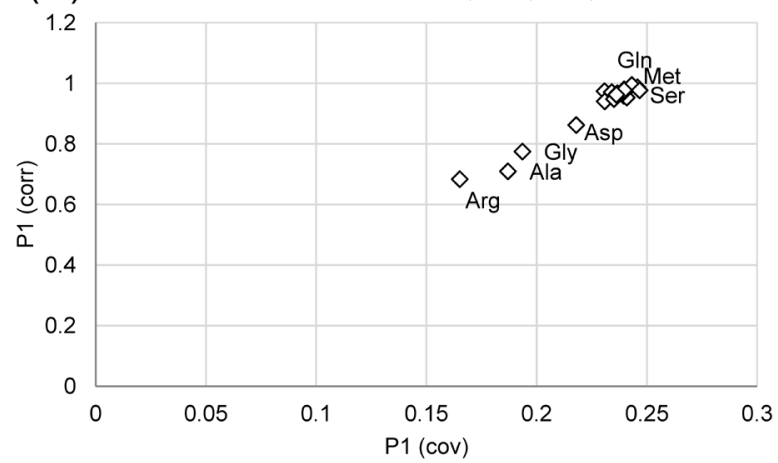

Fig. 7. S-Plots Obtained from Orthogonal Partial Least Squares Discriminant Analyses of Extracellular (A and B) and Intracellular (C and D) Amino Acids of PANC-1 Cells Treated with GEM under Normoglycemic Conditions (Upper Row) and PP under Hypoglycemic Conditions (Bottom Row)

P1 (cov) and P1 (corr) represent covariance and correlation of class predicted components, respectively.

pancreatic cancer compared to healthy controls. A study indicated that the level of five amino acids (Asn, Ile, Ala, His, and Trp) decrease and Ser levels increase in pancreatic cancer, allowing the identification of an aminoindex for early detection of pancreatic cancer. ${ }^{29)}$ In a different study, Nishiumi et al. indicated the increase of Glu and Asn and the decrease of Gly in pancreatic cancer. ${ }^{30)}$ Although some of the amino acids we identified in this study were also suggested as markers in previous reports, others are novel marker candidates that discriminate the efficacy of anticancer drugs. The discrepancies between different studies are probably due to differences in samples, preparation conditions, and analytical methods.

\section{DISCUSSION}

In this study, we evaluated the effects of the presence of glucose on the efficacy of anticancer drugs in PANC-1 cells, using intracellular and extracellular amino acid metabolomics. Resistance to GEM because of glucose depletion and PP selectivity under hypoglycemic conditions were confirmed by our score plots. Discrimination of anticancer effects was possible in the analysis of extracellular amino acids. Furthermore, analysis of intracellular amino acids was found to be a superior approach because the results obtained more clearly distinguished the cell states. Our amino acid metabolomics study allowed the identification of amino acid candidates that might be used as new biomarkers to evaluate the efficacy of anticancer drug. Cancer cells mainly produce energy via anaerobic glycolysis, even in an oxygen rich environment (the Warburg effect). ${ }^{31)}$ Glucose is converted to pyruvic acid by glycolytic enzymes, and then into lactic acid by lactic acid dehydrogenase. When cancer tissues or cells are exposed to hypoglycemic conditions, amino acids and fatty acids are used instead of glucose for energy production through the TCA cycle, which is suppressed because of the deficiency in intermediates under hypoglycemic conditions. For example, Gln is usually used as an alternative energy source (glutaminolysis). ${ }^{32)}$ Gln is converted via Glu to 2-oxoglutarate. Thus, the energy production system is shifted toward oxidative phosphorylation (OxPhos) by recruiting intermediates of the TCA cycle. Additionally, cancer tissues have increased amino acid concentrations compared with normal tissues. ${ }^{33,34)}$ This observation suggested that autophagy is induced to supplement amino acids for energy production under glucose starvation. ${ }^{35,36)}$ In the present study, the Gln concentration decreased sequentially in any condition, and we presumed it was being used for cell survival and proliferation. The content of almost amino acids in the cell extracts also increased when exposed to glucose starvation conditions (supplementary materials). These results are consistent with the reports by Kami et al. ${ }^{37}$ ) Further investigation using not only amino acids, but also TCA cycle intermediates as target analytes might provide more details regarding the metabolic alterations in cancer cells exposed to glucose starvation. Moreover, agents that overcome the tolerance of cancer cells to conditions found in the tumor microenvironment, such as nutrient starvation and hypoxia, have recently been explored as a novel approach in anticancer drug discovery. For example, (+)-grandifloracin, ${ }^{38)}$ kigamicin $\mathrm{D}^{39)}$ arctigenin, ${ }^{40)}$ and tirapazamine ${ }^{41)}$ show preferential toxicity to cancer cells under nutrient starvation or hypoxic 
conditions. Therefore, our proposed methods might be applicable for screening, evaluation of efficacy, and elucidation of mechanisms in the field of anticancer drug discovery research.

Conflict of Interest The authors declare no conflict of interest.

Supplementary Materials The online version of this article contains supplementary materials.

\section{REFERENCES}

1) Vinayavekhin N, Homan EA, Saghatelian A. Exploring disease through metabolomics. ACS Chem. Biol., 5, 91-103 (2010).

2) Ulaszewska MM, Trost K, Stanstrup J, Tuohy KM, Franceschi P, Chong M-F-F, George T, Minihane AM, Lovegrove JA, Mattivi F. Urinary metabolomic profiling to identify biomarkers of a flavonoid-rich and flavonoid-poor fruits and vegetables diet in adults: the FLAVURS trial. Metabolomics, 12, 32 (2016).

3) Nishiumi S, Kobayashi T, Ikeda A, Yoshie T, Kibi M, Izumi Y, Okuno T, Hayashi N, Kawano S, Takenawa T, Azuma T, Yoshida M. A novel serum metabolomics-based diagnostic approach for colorectal cancer. PLOS ONE, 7, e40459 (2012).

4) Yuliana ND, Khatib A, Verpoorte R, Choi YH. Comprehensive extraction method integrated with NMR metabolomics: a new bioactivity screening method for plants, adenosine A1 receptor binding compounds in Orthosiphon stamineus Benth. Anal. Chem., 83, 6902-6906 (2011).

5) Song Q, Chen H, Li Y, Zhou H, Han Q, Diao X. Toxicological effects of benzo $(\alpha)$ pyrene, DDT and their mixture on the green mussel Perna viridis revealed by proteomic and metabolomics approaches. Chemosphere, 144, 214-224 (2016).

6) Takahashi K, Kohno H. Different polar metabolites and protein profiles between high- and low-quality Japanese ginjo sake. PLOS $O N E$, 11, e0150524 (2016)

7) Inoue $\mathrm{K}$, Tanada $\mathrm{C}$, Sakamoto $\mathrm{T}$, Tsutsui H, Akiba T, Min JZ, Todoroki K, Yamano Y, Toyo'oka T. Metabolomics approach of infant formula for the evaluation of contamination and degradation using hydrophilic interaction liquid chromatography coupled with mass spectrometry. Food Chem., 181, 318-324 (2015).

8) Noguchi Y, Zhang QW, Sugimoto T, Furuhata Y, Sakai Y, Mori M, Takahashi M, Kimura T. Network analysis of plasma and tissue amino acids and the generation of an amino index for potential diagnostic use. Am. J. Clin. Nutr., 83, 513S-519S (2006).

9) Zhang Q, Takahashi M, Noguchi Y, Sugimoto T, Kimura T, Okumura A, Ishikawa T, Kakumu S. Plasma amino acid profiles applied for diagnosis of advanced liver fibrosis in patients with chronic hepatitis C infection. Hepatol. Res., 34, 170-177 (2006).

10) Kimura $T$, Hamase $K$, Miyoshi Y, Yamamoto R, Yasuda K, Mita M, Rakugi H, Hayashi T, Isaka Y. Chiral amino acid metabolomics for novel biomarker screening in the prognosis of chronic kidney disease. Sci. Rep., 6, 26137 (2016).

11) Kalíková K, Slechtová T, Tesařová E. Enantiomeric ratio of amino acids as a tool for determination of aging and disease diagnostics by chromatographic measurement. Separations, 3, 30 (2016).

12) Mochizuki T, Takayama T, Todoroki K, Inoue K, Min JZ, Toyo'oka T. Towards the chiral metabolomics: liquid chromatography-mass spectrometry based DL-amino acid analysis after labeling with a new chiral reagent, $(S)$-2,5-dioxopyrrolidin-1-yl-1-(4,6-dimethoxy1,3,5-triazin-2-yl)pyrrolidine-2-carboxylate, and the application to saliva of healthy volunteers. Anal. Chim. Acta, 875, 73-82 (2015).

13) Miyagi Y, Higashiyama M, Gochi A, Akaike M, Ishikawa $T$, Miura T, Saruki N, Bando E, Kimura H, Imamura F, Moriyama M, Ikeda I, Chiba A, Oshita F, Imaizumi A, Yamamoto H, Miyano H, Horimoto K, Tochikubo O, Mitsushima T, Yamakado M, Okamoto
N. Plasma free amino acid profiling of five types of cancer patients and its application for early detection. PLoS ONE, 6, e24143 (2011).

14) Simińska E, Koba M. Amino acid profiling as a method of discovering biomarkers for early diagnosis of cancer. Amino Acids, $\mathbf{4 8}$, $1339-1345$ (2016)

15) Tomita R, Todoroki K, Machida K, Nishida S, Maruoka H, Yoshida H, Fujioka T, Nakashima M, Yamaguchi M, Nohta H. Assessment of the efficacy of anticancer drugs by amino acid metabolomics using fluorescence derivatization-HPLC. Anal. Sci., 30, 751-758 (2014).

16) Tomita R, Todoroki K, Maruoka H, Yoshida H, Fujioka T, Nakashima M, Yamaguchi M, Nohta H. Amino acid metabolomics using LC-MS/MS: Assessment of cancer-cell resistance in a simulated tumor microenvironment. Anal. Sci., 32, 893-900 (2016).

17) Onozuka H, Tsuchihara K, Esumi H. Hypoglycemic/hypoxic condition in vitro mimicking the tumor microenvironment markedly reduced the efficacy of anticancer drugs. Cancer Sci., 102, 975-982 (2011).

18) Grasso C, Jansen G, Giovannetti E. Drug resistance in pancreatic cancer: impact of altered energy metabolism. Crit. Rev. Oncol. Hematol., 114, 139-152 (2017).

19) Valenzuela MMA, Neidigh JW, Wall NR. Antimetabolite treatment for pancreatic cancer. Chemotherapy, 3, 137 (2014). doi: 10.4172/2167-7700.1000137

20) Heinemann V, Xu Y-Z, Chubb S, Sen A, Hertel LW, Grindey GB, Plunkett W. Inhibition of ribonucleotide reduction in CCRF-CEM cells by $2^{\prime}, 2^{\prime}$-difluorodeoxycytidine. Mol. Pharmacol., 38, 567-572 (1990).

21) Tomitsuka E, Kita K, Esumi H. The NADH-fumarate reductase system, a novel mitochondrial energy metabolism, is a new target for anticancer therapy in tumor microenvironments. Ann. N.Y. Acad. Sci., 1201, 44-49 (2010).

22) Esumi H, Lu J, Kurashima Y, Hanaoka T. Antitumor activity of pyrvinium pamoate, 6-(dimethylamino)-2-[2-(2,5-dimethyl1-phenyl-1 H-pyrrol-3-yl)ethenyl]-1-methyl-quinolinium pamoate salt, showing preferential cytotoxicity during glucose starvation. Cancer Sci., 95, 685-690 (2004).

23) Tomitsuka E, Kita K, Esumi H. An anticancer agent, pyrvinium pamoate inhibits the NADH-fumarate reductase system - a unique mitochondrial energy metabolism in tumor microenvironments. $J$. Biochem., 152, 171-183 (2012).

24) Ishii I, Harada Y, Kasahara T. Reprofiling a classical anthelmintic, pyrvinium pamoate, as an anti-cancer drug targeting mitochondrial respiration. Front. Oncol., 2, 137 (2012).

25) Ishiyama M, Miyazono Y, Sasamoto K, Ohkura Y, Ueno K. A highly water-soluble disulfonated tetrazolium salt as a chromogenic indicator for NADH as well as cell viability. Talanta, 44, 1299-1305 (1997).

26) Daga RR, Bolaños P, Moreno S. Regulated mRNA stability of the Cdk inhibitor Rum1 links nutrient status to cell cycle progression. Curr. Biol., 13, 2015-2024 (2003).

27) Gabai VL, Zamulaeva IV, Mosin AF, Makarova YM, Mosina VA, Budagova KR, Malutina YV, Kabakov AE. Resistance of Ehrlich tumor cells to apoptosis can be due to accumulation of heat shock proteins. FEBS Lett., 375, 21-26 (1995).

28) Akakura N, Kobayashi M, Horiuchi I, Suzuki A, Wang J, Chen J, Niizeki H, Kawamura K, Hosokawa M, Asaka M. Constitutive expression of hypoxia-inducible factor-1alpha renders pancreatic cancer cells resistant to apoptosis induced by hypoxia and nutrient deprivation. Cancer Res., 61, 6548-6554 (2001).

29) Fukutake N, Ueno M, Hiraoka N, Shimada K, Shiraishi K, Saruki $\mathrm{N}$, Ito T, Yamakado M, Ono N, Imaizumi A, Kikuchi S, Yamamoto $\mathrm{H}$, Katayama K. A novel multivariate index for pancreatic cancer detection based on the plasma free amino acid profile. PLOS ONE, 10, e0132223 (2015)

30) Nishiumi S, Shinohara M, Ikeda A, Yoshie T, Hatano N, Kakuyama 
S, Mizuno S, Sanuki T, Kutsumi H, Fukusaki E, Azuma T, Takenawa T, Yoshida M. Serum metabolomics as a novel diagnostic approach for pancreatic cancer. Metabolomics, 6, 518-528 (2010).

31) Warburg O. On the origin of cancer cells. Science, 123, 309-314 (1956).

32) DeBerardinis RJ, Mancuso A, Daikhin E, Nissim I, Yudkoff M, Wehrli S, Thompson CB. Beyond aerobic glycolysis: transformed cells can engage in glutamine metabolism that exceeds the requirement for protein and nucleotide synthesis. Proc. Natl. Acad. Sci. U.S.A., 104, 19345-19350 (2007).

33) Hirayama A, Kami K, Sugimoto M, Sugawara M, Toki N, Onozuka H, Kinoshita T, Saito N, Ochiai A, Tomita M, Esumi H, Soga T. Quantitative metabolome profiling of colon and stomach cancer microenvironment by capillary electrophoresis time-of-flight mass spectrometry. Cancer Res., 69, 4918-4925 (2009).

34) Zhang J, Liu L, Wei S, Nagana Gowda GA, Hammoud Z, Kesler KA, Raftery D. Metabolomics study of esophageal adenocarcinoma. J. Thorac. Cardiovasc. Surg., 141, 469-475, 475.e1-475.e4 (2011).

35) Sato K, Tsuchihara K, Fujii S, Sugiyama M, Goya T, Atomi Y, Ueno T, Ochiai A, Esumi H. Autophagy is activated in colorectal cancer cells and contributes to the tolerance to nutrient deprivation. Cancer Res., 67, 9677-9684 (2007).

36) Hashimoto D, Bläuer M, Hirota M, Ikonen NH, Sand J, Laukkari- nen J. Autophagy is needed for the growth of pancreatic adenocarcinoma and has a cytoprotective effect against anticancer drugs. Eur. J. Cancer, 50, 1382-1390 (2014).

37) Kami K, Tomitsuka E, Tomita M, Kita K, Esumi H, Soga T. Elucidation of energy metabolism of cancer microenvironment by metabolome analysis. Experimental medicine Extra. (Esumi H, Takakura N, Miyazono K, Mori M. ed.) Vol. 27, Yodosha, Tokyo, Japan, pp. 215-221 (2009).

38) Ueda J-Y, Athikomkulchai S, Miyatake R, Saiki I, Esumi H, Awale S. (+)-Grandifloracin, an antiausterity agent, induces autophagic PANC-1 pancreatic cancer cell death. Drug Des. Devel. Ther., 8, 39-47 (2013).

39) Lu J, Kunimoto S, Yamazaki Y, Kaminishi M, Esumi H. Kigamicin $\mathrm{D}$, a novel anticancer agent based on a new anti-austerity strategy targeting cancer cells' tolerance to nutrient starvation. Cancer Sci., 95, 547-552 (2004).

40) Awale S, Lu J, Kalauni SK, Kurashima Y, Tezuka Y, Kadota S, Esumi H. Identification of arctigenin as an antitumor agent having the ability to eliminate the tolerance of cancer cells to nutrient starvation. Cancer Res., 66, 1751-1757 (2006).

41) Peters KB, Brown JM. Tirapazamine: a hypoxia-activated topoisomerase II poison. Cancer Res., 62, 5248-5253 (2002). 